\title{
Bronchoskopische Lungenvolumenreduktion bei fortgeschrittenem Lungenemphysem: Zukunftsmusik oder viel Lärm um nichts?
}

\section{Bronchoscopic Lung Volume Reduction (BLVR) in Advanced Pulmonary Emphysema: Dreams of the Future or Much Ado About Nothing?}

Autor

Institut

\author{
F. Stanzel
}

Pneumologische Abteilung, Lungenklinik Hemer, Hemer (Chefärzte: Dr. M. Serke, Dr. F. Stanzel, Dr. M. Westhoff) eingereicht 11.9. 2011 akzeptiert nach Revision 28. 9. 2011

\section{Bibliografie}

DOI http://dx.doi.org/ 10.1055/s-0031-1291458 Online-Publikation: 23.11.2011 Pneumologie 2012; 66: 20-27 (c) Georg Thieme Verlag KG Stuttgart · New York ISSN 0934-8387

Korrespondenzadresse Dr. Franz Stanzel Pneumologische Abteilung Lungenklinik Hemer Theo-Funccius-Str. 1 58675 Hemer franz.stanzel@lkhemer.de

\section{Zusammenfassung}

$\nabla$

Die endoskopische Lungenvolumenreduktion (ELVR) entwickelt sich rasch weiter und wird derzeit intensiv untersucht und diskutiert. Der Ansatz, eine Lungenvolumenreduktion zu erreichen, ohne die Risiken eines chirurgischen Eingriffs eingehen zu müssen, ist sehr vielversprechend. Die verschiedenen Techniken der ELVR sind durch geringere Morbidität und Mortalität gekennzeichnet. Allerdings ist der erzielte Effekt meist eher limitiert. Die Blockade von Atemwegen durch Ventile mit dem Ziel einer Reduktion des Lungenvolumens durch Ausbildung einer Absorptionsatelektase ist bislang am intensivsten untersucht und angewandt worden. Den blockierenden Verfahren stehen teilblockierende Verfahren wie die Einlage von Coils und nicht blockierende irreversible Verfahren wie die polymerische Lungenvolumenreduktion oder die thermische Ablation gegenüber. Die Schaffung von Atemwegsbypässen, die vorwiegend beim homogenen Emphysem eingesetzt wird, führt vorübergehend zur Verbesserung von Lungenfunktionsparametern und zur Reduktion von Dyspnoe. Letztlich ist die erzielte Verbesserung aber von kurzer Dauer und hat sich deshalb nicht durchgesetzt. Diese Arbeit soll einen Überblick über den derzeitigen Stand des Wissens zur ELVR geben.

\section{Einleitung}

$\nabla$

Die chronisch obstruktive Lungenerkrankung (COPD) ist eine der verbreitetsten Erkrankungen überhaupt und nimmt an Häufigkeit zu. Es handelt sich um eine progressive Erkrankung, die durch irreversible Überblähung und Zerstörung der Lungenabschnitte distal der Bronchioles terminales charakterisiert ist. Es kommt zu einem mehr oder minder ausgeprägten Emphysem. Eine nicht reversible Atemwegsobstruktion, eine Reduktion der Oberfläche für den Gasaustausch und eine Re-

\section{Abstract \\ $\nabla$}

Bronchoscopic lung volume reduction (BLVR) is a rapidly developing area and at present it is being intensively evaluated and discussed. There is a great interest in developing new treatment modalities that can reduce lung volume and air trapping without the risk of a surgical intervention. The different techniques of BLVR are characterised by lower morbidity and mortality, but by a more limited effect too. The placement of valves leads to blockade of the airway and sometimes to absorption atelectasis. The valves have been most intensively evaluated and are frequently applied. Beside the blocking devices there are partially blocking or deforming devices available as coils that are introduced in heterogeneous emphysema. Irreversible procedures such as polymeric lung volume reduction or thermal vapour ablation are used too. The creation of airway bypasses to allow trapped air to escape is mainly employed in homogenous emphysema. Following such bypass creation there is an increase of lung function tests and a reduction of dyspnea, but only for a limited time. The bypass procedure has disappeared from bronchoscopy units completely. We give a review of the recent developments regarding BLVR and the state of the art.

duktion der Lungenelastizität sind weitere Merkmale. Es besteht eine zunehmende Instabilität der kleinen Atemwege mit exspiratorischem Kollaps. Statische und dynamische Überblähung sind die Folgen, das typische Leitsymptom ist eine progrediente Belastungsdyspnoe. Die Standardtherapie umfasst Bronchodilatatoren, Steroide, Belastungstraining und/oder Rehabilitationsprogramme und wenn erforderlich Sauerstoff. Trotz dieser Therapie bleiben die Patienten aber in den fortgeschrittenen Stadien der Erkrankung erheblich eingeschränkt [1]. Die Prognose ist schlecht. 


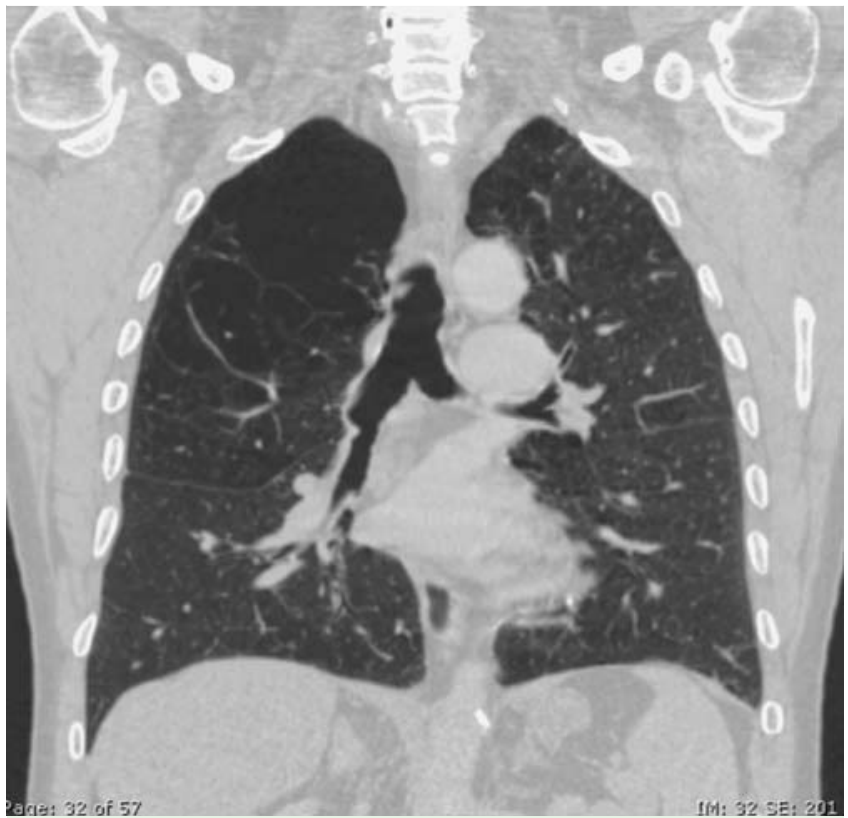

Abb. 1 Koronare Rekonstruktion des HR-CT mit typischem oberlappenbetontem Emphysem, das rechts deutlich ausgeprägter imponiert.

Bereits in den 1950er-Jahren wurde die chirurgische Volumenreduktion entwickelt. Die hohe peri- und postoperative Mortalität führte dazu, dass sich das Verfahren trotz positiver postoperativer Ergebnisse nicht verbreitete. In den 1990er-Jahren wurde das Prinzip wieder aufgegriffen. Durch die Verminderung der Hyperinflation wird die elastische Rückstellkraft der Lungen verbessert und die Atemmechanik optimiert. Das führt zur Reduktion von Atemnot, zur Verbesserung der Belastungsfähigkeit und Lebensqualität bei selektionierten Patientengruppen [2-6]. Die NETT-Studie (National Emphysema Treatment Trial [3]), eine randomisierte multizentrische US-amerikanische Studie, vergleicht die bilaterale chirurgische Lungenvolumenreduktion mit der rein medikamentösen Therapie (608 vs. 610 Patienten). Bei Patienten mit oberlappenbetontem Lungenemphysem ( $\mathbf{A b b} \mathbf{1}$ ) zeigt sich sowohl eine Verbesserung der Lungenfunktion als auch der Lebensqualität. Die Gruppe der operierten Patienten weist allerdings eine erheblich höhere 90-Tage-Sterblichkeit auf, verglichen mit der Gruppe der konservativ behandelten Patienten $(7,9 \%$ vs. $1,3 \%)$. Diese ist hauptsächlich durch Patienten mit erheblich eingeschränkter Funktion $\left(\mathrm{FEV}_{1}<20 \%\right.$, Diffusionskapazität $<20 \%$ ) oder homogenem Emphysem bedingt. Andere Probleme sind hohe Kosten und vornehmlich die hohe Rate an persistierenden Fisteln, die eine Verlängerung der stationären Verweildauer, eine höhere Morbidität und hohe frühe Mortalität bedingt $[2,3,5,6]$.

Die endoskopische Lungenvolumenreduktion (ELVR) hat als möglicherweise sicherere Behandlungsalternative zwischenzeitlich an Bedeutung gewonnen und kommt auch in Betracht, wenn die chirurgische Lungenvolumenreduktion nicht möglich ist [1]. Sie wird derzeit intensiv untersucht und diskutiert $[1,7,8]$. Die Verfahren sind durch eine geringere Morbidität und Mortalität gekennzeichnet [9]. Durch verschiedene endoskopische Verfahren wird versucht, eine Reduktion der Überblähung zu erreichen. Es ist zwischen reversiblen, teil-reversiblen und irreversiblen Verfahren zu unterscheiden ( Tab.1). Ferner können die verschiedenen Verfahren entweder mehr dem heterogenen oder dem homogenen Emphysem zugeordnet werden mit der Aus-
Tab. 1 Diverse Verfahren der Lungenvolumenreduktion.

\begin{tabular}{|c|c|c|c|}
\hline Verfahren & Charakteristik & & $\begin{array}{l}\text { Emphysem- } \\
\text { typ }\end{array}$ \\
\hline $\begin{array}{l}\text { Endobronchiale } \\
\text { Ventile }\left(Z_{\text {ephyr }}^{\circledR}\right)\end{array}$ & blockierend & reversibel & heterogen \\
\hline $\begin{array}{l}\text { Intrabronchiale } \\
\text { Ventile (Spiration }{ }^{\circledR} \text { ) }\end{array}$ & blockierend & reversibel & heterogen \\
\hline Coils (PneumRx ${ }^{\circledR}$ ) & $\begin{array}{l}\text { nicht- } \\
\text { blockierend }\end{array}$ & $\begin{array}{l}\text { teil-oder } \\
\text { irreversibel }\end{array}$ & heterogen \\
\hline Polymer (AeriSeal ${ }^{\circledR}$ ) & $\begin{array}{l}\text { nicht- } \\
\text { blockierend }\end{array}$ & irreversibel & $\begin{array}{l}\text { hetero- und } \\
\text { homogen }\end{array}$ \\
\hline Dampf (Uptake ${ }^{\circledR}$ ) & $\begin{array}{l}\text { nicht- } \\
\text { blockierend }\end{array}$ & irreversibel & heterogen \\
\hline Bypässe (Broncus ${ }^{\circledR}$ ) & $\begin{array}{l}\text { (nicht- } \\
\text { blockierend) }\end{array}$ & $\begin{array}{l}\text { teilreversibel, } \\
\text { reversibel? }\end{array}$ & homogen \\
\hline $\begin{array}{l}\text { Pneumostoma } \\
\left(\text { Portaero }^{\circledR}\right)\end{array}$ & $\begin{array}{l}\text { (nicht- } \\
\text { blockierend) }\end{array}$ & $\begin{array}{l}\text { teilreversibel, } \\
\text { reversibel? }\end{array}$ & homogen \\
\hline
\end{tabular}

nahme der polymerischen Lungenvolumenreduktion, die sowohl beim homogenen als auch beim heterogenen Emphysem eingesetzt werden kann ( $\bullet$ Tab.1). Bislang fehlen noch weitgehend Daten zu den Langzeitergebnissen dieser Techniken [1].

\section{Anamnese, Grunduntersuchungen}

Anamnese

Wichtige Schlüsselsymptome sind die Ausprägung der Atemnot, insbesondere die Belastungsfähigkeit und die Häufigkeit von Infekten. Nur fortgeschrittene Stadien der COPD mit mehr oder minder ausgeprägter Belastungsatemnot kommen für eine ELVR in Frage. Große Mengen von Sputum, eitriges Sputum, häufige Infekte sprechen gegen eine ELVR oder machen diese schwieriger. Bronchiektasen sind in der Regel ein Ausschlusskriterium. Vor einer ELVR ist es essenziell, dass der Patient den Zigarettenkonsum eingestellt hat. Meist wird eine Nikotinkarenz von mindestens 3 Monaten vor einem solchen Eingriff gefordert.

\section{Basisuntersuchungen}

$\mathrm{Zu}$ den Basisuntersuchungen zählt die Erfassung der Lungenfunktion. In einer Bodyplethysmografie nach Bronchospasmolyse gelten der $\mathrm{FEV}_{1}<45 \%$ und das Residualvolumen $>150 \%$, bezogen auf den Vorhersagewert, als Grenzen, ab denen eine ELVR indiziert sein kann. Ein $\mathrm{FEV}_{1}<20 \%$ bedeutet ein erhöhtes Risiko, sodass diese Patienten in den meisten Studien ausgeschlossen werden. Die Diffusionskapazität muss erfasst werden. Werte unter 20\% bedeuten einen Anstieg des Risikos, weil sie Indikator für eine weit fortgeschrittene Erkrankung sind. Daneben muss die Blutgasanalyse durchgeführt werden. Ein $\mathrm{pO}_{2}>45$ Torr und ein $\mathrm{pCO}_{2}<50$ Torr gelten als Grenzen für eine ELVR. Auch sollte die Belastungsfähigkeit vor Durchführung einer ELVR objektiv erfasst werden. Dies kann am einfachsten über einen standardisierten 6Minuten-Gehtest erfolgen. Dabei gilt eine Gehstrecke von > $140 \mathrm{~m}$ als Grenzwert. Alternativ kann natürlich eine Fahrradergometrie mit Blutgasanalyse oder eine Ergospirometrie durchgeführt werden.

Die Computertomografie der Lunge in hochauflösender Technik ist ganz wesentlich, weil damit das Emphysem charakterisiert wird und die möglichen Zielregionen für die Therapie bestimmt werden. Dies kann im Rahmen einer visuellen Beurteilung der Schnitte erfolgen ( $\bullet$ Abb. 2 a). Ein sogenannter Heterogenitätsin- 

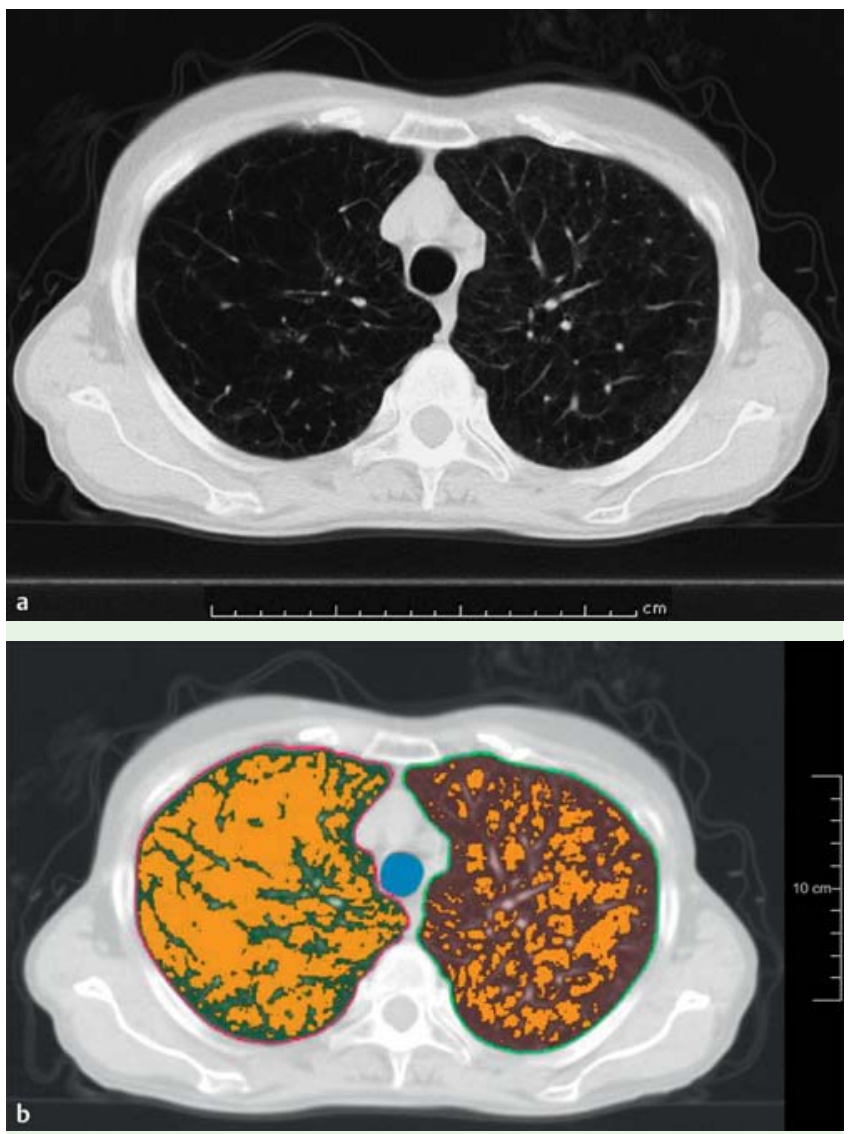

Abb.2 a Heterogenes Lungenemphysem in der HR-CT mit deutlicher Betonung des rechten Oberlappens. b Die farbliche Markierung vereinfacht wesentlich die Erfassung und die Abschätzung des Schweregrades der Destruktion (MeVisPULMO ${ }^{\circledR}$ ).

dex, der die Dichteunterschiede zahlenmäßig widerspiegelt, erlaubt eine objektivere Einschätzung. Rekonstruktionen des CT mit sagittalen oder koronaren Schnitten sind bei der Beurteilung hilfreich. Der Einsatz einer speziellen Software, die emphysematöse Areale anhand eines vorgegebenen Dichtegrenzwertes farblich markiert darstellt, erleichtert die visuelle Auswertung (beispielsweise YACTRA ${ }^{\circledast}$, MeVisPULMO $^{\circledR}[10], \odot$ Abb.2 b). Größere Bullae von mehr als $1 / 3$ des Hemithorax sind abzugrenzen, da sie in der Regel eine Kontraindikation zur ELVR darstellen.

Die Perfusions-Szintigrafie erlaubt eine Beurteilung der funktionellen Relevanz der visualisierten Veränderungen. Die Standardtechnik umfasst die Darstellung in 6 Projektionen, die weniger aufwändig und in aller Regel ausreichend ist ( $\bullet$ Abb. 3). Wenn die Szintigrafie in SPECT-Technik durchgeführt wird, ist die Zuordnung exakter. Allerdings bedeutet dies einen zusätzlichen erheblichen Arbeitsaufwand. Areale mit einer reduzierten Perfusion, die in der CT eine stärkere emphysematöse Umwandlung aufweisen, stellen die möglichen Zielregionen für eine ELVR dar.

\section{Die verschiedenen Verfahren}

$\nabla$

Für das heterogene Emphysem stehen blockierende und nichtblockierende Verfahren zur Verfügung. Ein Teil hat auch schon ein CE-Zertifikat für den europäischen Markt.

\section{Blockierende Verfahren}

Das Grundprinzip der blockierenden Verfahren stellt ein EinWeg-Ventil dar [11]. Dieses verhindert das Einströmen von Atemluft während der Inspiration über den Bronchus, weil er blockiert ist. Bei der Exspiration kommt aber der Ventilmechanismus zum Tragen und erlaubt den Austritt von Luft und auch von Sekreten. Dadurch kommt es zur Reduktion der Überblähung bis hin zum Auftreten einer Absorptionsatelektase. Dann ist in der Regel der größte Volumenreduktionseffekt zu erreichen. Ventile werden relativ zentral im Segment- oder Subsegmentbronchus nach einer Größenabschätzung über den Arbeitskanal eines flexiblen Bronchoskops platziert. Sieht man von bisweilen auftretenden Granulationen ab, ist die Ventilimplantation grundsätzlich als reversibel einzuschätzen, weil diese auch bronchoskopisch erreicht und gegebenenfalls wieder entfernt werden können.

Es sind 2 verschiedene Typen von Ventilen für die Atemwege erhältlich. Sogenannte endobronchiale Ventile (EBV, Zephyr ${ }^{\circledR}$, Pulmonx, USA, $\odot$ Abb.4a) unterscheiden sich nicht in der Funktionsweise von intrabronchialen Ventilen (IBV, Spiration ${ }^{\circledR}$, Olympus, USA, $\odot$ Abb.4b, $\odot$ Video 1). Beide werden mittels eines jeweils speziellen Applikationssystems bronchoskopisch eingesetzt. Der Arbeitskanal muss mindestens $2,6 \mathrm{~mm}$ betragen.

Das Zephyr-Ventil besteht aus Silikon und einem Nitinol-Gerüst. Das Ventil ist fischmaulartig konfiguriert. Es ist in 2 Größen erhältlich. Die Größe wird mit dem Applikationskatheter anhand eines kleinsten und größten geeigneten Durchmessers abgeschätzt. Die Implantation der Zephyr-Ventile führt zu einer Zunahme des $\mathrm{FEV}_{1}$ und der Diffusionskapazität. CT-morphologisch kommt es zu einem Rückgang der Lungenblähung. Zudem zeigten Befindlichkeits-Scores eine Verbesserung. Die Ergebnisse einer Pilotstudie und von mehreren Folgestudien wurden ab 2003 publiziert $[12,13]$. Im Rahmen der prospektiven multizentrischen randomisierten VENT-Studie (Endobronchial Valve for Emphysema Palliation Trial) wird die Sicherheit und Effektivität der Therapie mit Ventilen überprüft und mit einer konservativen Standardtherapie verglichen [7]. Eingeschlossen sind 321 Patienten, 220 mit unilateraler Ventilimplantation, 101 Patienten mit leitliniengerechter konservativer Therapie. Der $\mathrm{FEV}_{1}$ ist in der mit Ventilen therapierten Gruppe um 6,8\% größer, der 6-Minuten-Gehtest um 5,8\% verbessert. Diese Parameter stellen primäre Endpunkte dar. Die Verbesserung ist zwar signifikant, allerdings zahlenmäßig relativ gering. Das 12-Monats-Follow-up ergibt eine höhere Komplikationsrate in der mit Ventilen behandelten Patientengruppe (10,2\% vs. 4,6\%). Allerdings ergeben sich keine Unterschiede in der Mortalität oder in der Pneumonie-Rate, bezogen auf den behandelten Lungenlappen. Ein wichtiger Punkt: Die Patienten mit dem höchsten Heterogenitätsindex hatten den größten Nutzen.

Bei Subgruppenanalysen lassen sich weitere Faktoren für einen Erfolg der Ventilimplantation herausarbeiten. Ganz wesentlich ist die exakte Einlage der Ventile. Der Lappen muss vollständig
Video 1

Liegendes Spiration-Ventil im benachbarten Subsegmentbronchus. Der Katheter mit dem geladenen Schirmchen wird vorgeführt. Dann wird dieses an geeigneter Stelle freigesetzt.

Video unter:

www.thieme-connect.de/ejournals/doi/10.1055/s-0031-1291458 


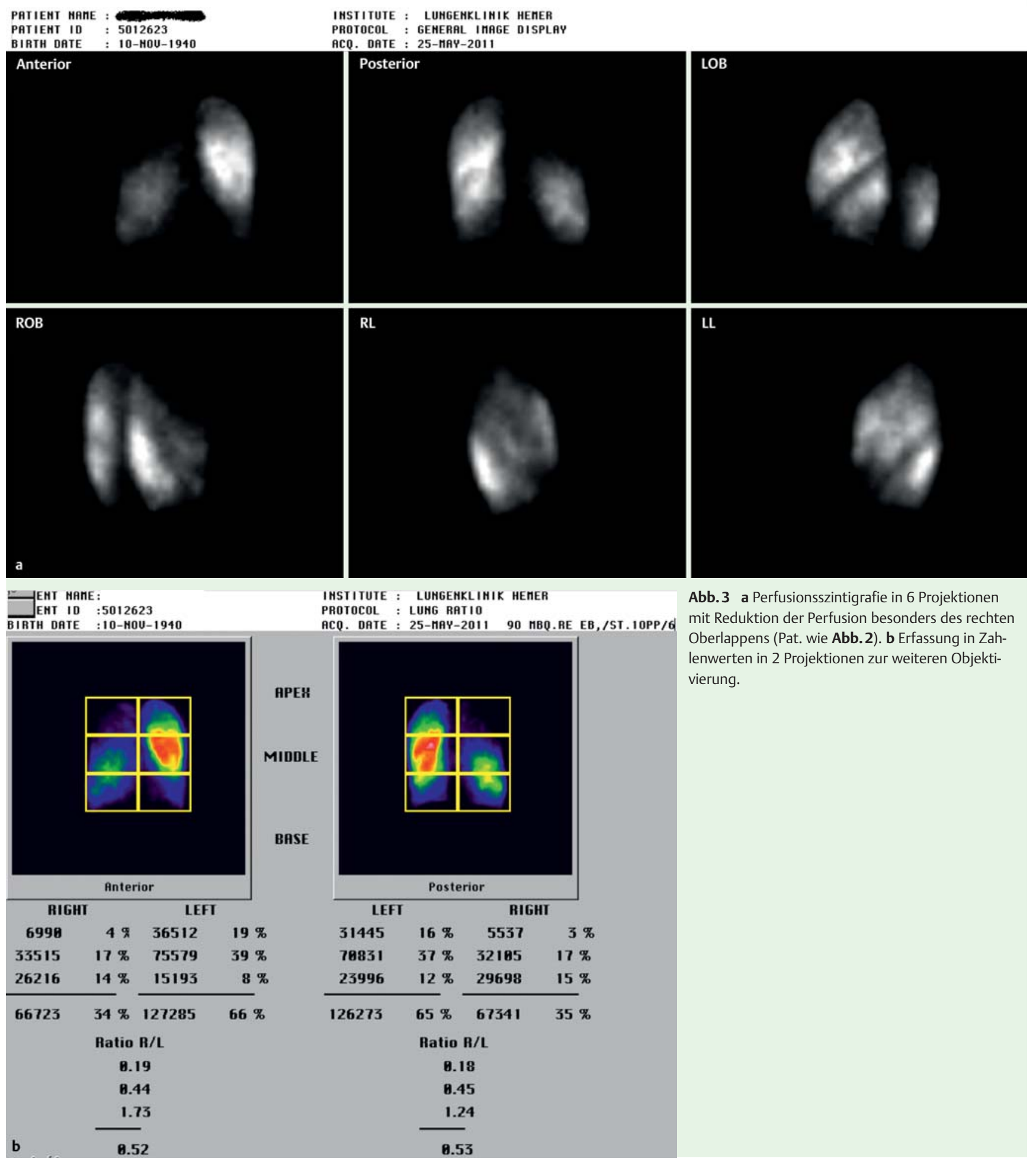

verschlossen sein. Nur dann kann sich im Idealfall eine Atelektase entwickeln. Patienten mit unvollständigem Verschluss zeigen schlechtere Ergebnisse [7].

Subgruppenanalysen zeigen, dass bei Patienten, bei denen die Rekonstruktionen der CT vollständige intakte Fissuren nachweisen, bessere Ergebnisse nach Ventilimplantation gefunden werden. Die Fissuren werden auf koronaren und sagittalen Rekonstruktionen der CT visuell erfasst ( Abb. 5). Vollständige Fissuren gehen mit hoher Wahrscheinlichkeit mit einer geringen oder nicht vorhandenen Kollateralventilation einher. Bei einem ausgeprägten Emphysem ist die Zahl kollateraler Kanäle erhöht.
Damit kann es selbst bei korrekter Lage von Ventilen und vollständigem Verschluss des Lappens zur retrograden Füllung mit Luft kommen und sich gegebenenfalls keine Absorptionsatelektase bilden.

Zwischenzeitlich steht ein Verfahren zur Verfügung, das die Messung der kollateralen Ventilation erlaubt. Diese wird bronchoskopisch mithilfe eines speziellen Ballonmesskatheters durchgeführt (Chartis ${ }^{\circledR}$, Pulmonx, USA). Dabei werden der Fluss und Druck in einem blockierten Lungenabschnitt gemessen. Daraus kann man auf den kollateralen Widerstand zurückschließen. In einer Pilotstudie zeigt sich, dass bei $90 \%$ der Patienten eine Übereinstim- 


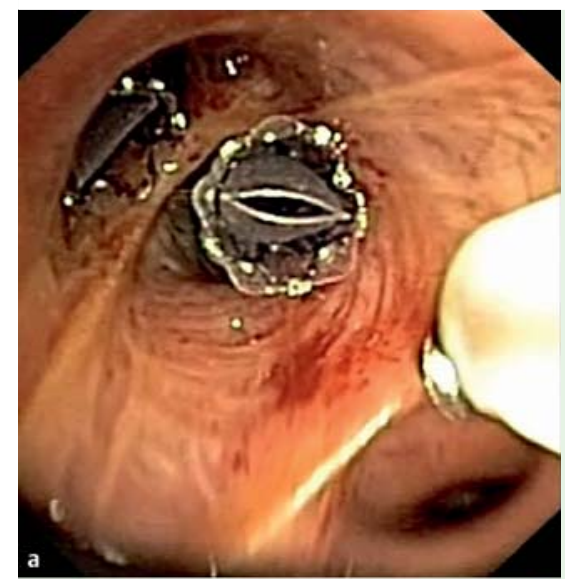

Abb.4 a Liegende EBVVentile (Zephyr $\left.{ }^{\circledR}\right)$, noch erkennbarer Applikationskatheter. Das Zentrale öffnet sich fischmaulartig. $\mathbf{b}$ Liegendes IBV-Ventil (Spiration ${ }^{\circledR}$ ), daneben gerade freigesetztes Ventil mit Applikationskatheter, der durch den Arbeitskanal des Bronchoskops eingeführt ist.

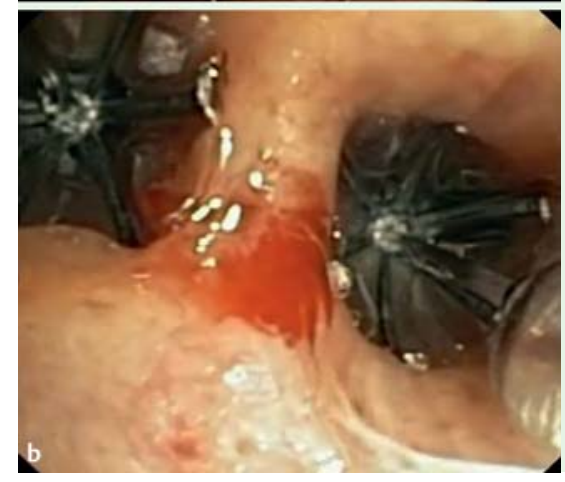

mung zwischen der Messung einer geringen kollateralen Ventilation und dem Nachweis von kompletten Fissuren in der Rekonstruktion der CT besteht [14]. Eine multizentrische Folgestudie soll nun klären, ob das Verfahren zur Patientenselektion geeignet ist.

Die intrabronchialen Ventile (IBV, Spiration ${ }^{\circledR}$ ) sind wie kleine Schirme gestaltet ( Abb. 4). Vom zentralen Bronchus aus schaut man in den geöffneten Schirm. Der Ventilmechanismus beruht auf einer Einfaltung des Schirms. Dadurch können Luft und Sekrete entweichen. Bei der Inspiration lässt dieser jedoch aufgrund der kompletten Ausfüllung der bronchialen Oberfläche keine Luft in den entsprechenden Lungenabschnitt. Distal sind kleine Haken angebracht, die sich in der Bronchialschleimhaut verankern und eine Migration verhindern.

Eine erste Studie zeigt bei 30 Patienten mit oberlappenbetontem Emphysem lediglich bezogen auf ein Befindlichkeitsquestionaire eine signifikante Verbesserung [15]. Die größere multizentrische Folgestudie an 91 Patienten, die in der Mehrzahl bilateral behandelt wurden, zeigt eine signifikante Verbesserung der Lebensqualität und eine Reduktion des Lungenvolumens in der Computertomografie [16]. Die Rate an Pneumothoraces ist bei komplettem Verschluss erhöht, sodass die meisten Patienten bilateral und ohne kompletten Verschluss behandelt wurden [17]. Dabei wird jeweils ein Subsegment des Oberlappens unverschlossen gelassen. Die Wirkungsweise der Lungenvolumenreduktion wird damit erklärt, dass es zu einer Umleitung des Atemstroms in funktionell bessere Areale kommt.

Allerdings sprechen zwischenzeitlich auch andere Daten für einen kompletten einseitigen Verschluss $[1,18]$, der durch die eingesetzten Ventile erreicht werden soll. Eberhardt et al. vergleichen die unilaterale mit der bilateralen endoskopischen Lungenvolumenreduktion. In die prospektive und randomisierte Studie sind 20 Patienten eingeschlossen. Damit ist die Patientenzahl allerdings vergleichsweise klein. Bei unilateralem komplettem Verschluss eines Oberlappens ( $\mathbf{A}$ bb. $\mathbf{6}$ ) ergibt sich bezüglich des $\mathrm{FEV}_{1}$ und des 6-Minuten-Gehtestes ein signifikant besseres Ergebnis, verglichen zum bilateralen Vorgehen (+32,5\% vs. $+2,5 \%$ bzw. $+43 \mathrm{~m}$ vs. $-19 \mathrm{~m}$ ). Eine andere Studie zeigt, dass bei Patienten, die nach ELVR mit Emphasys ${ }^{\circledR}$-Ventilen eine komplette Atelektase entwickeln, im Langzeitverlauf ein Überlebensvorteil nachzuweisen ist [1], der nicht auf Unterschiede in den Baseline-Charakteristika zurückgeführt werden kann. 5 von 19 Patienten entwickelten eine Atelektase nach Ventilimplantation. Keiner dieser Patienten ist im Langzeitverlauf bis 2010 verstorben, hingegen 8 von 14 Patienten, die keine Atelektase entwickelten.

\section{Teilblockierende und nicht blockierende Verfahren}

Die Einlage von Coils (PneumRx ${ }^{\circledR}$, Mountain View, USA) in einen Lungenabschnitt führt zu einer mechanischen Verformung mit Schrumpfung und Volumenreduktion. Diese Coils sind aus Nitinol gefertigt und werden in gestrecktem Zustand über einen
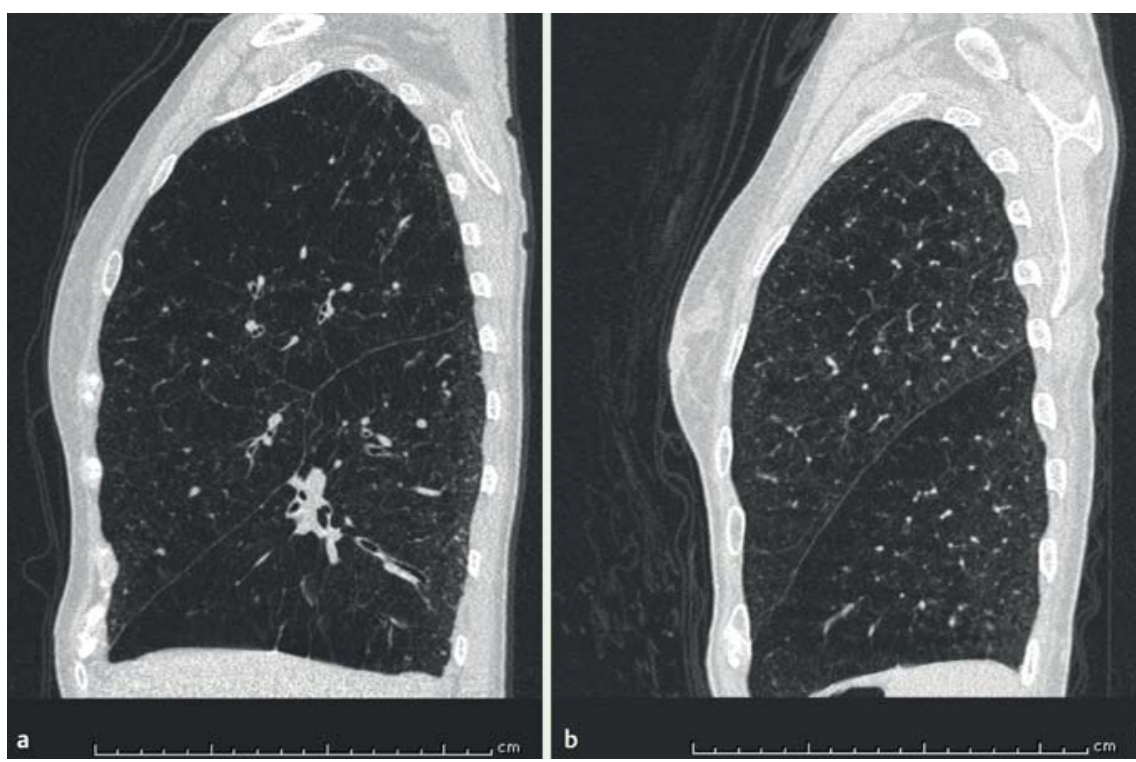

Abb. 5 a Sagittale Rekonstruktion rechts mit fast vollständig fehlender Fissur zwischen Ober- und Mittellappen, intakter Fissur zum Unterlappen (Pat. wie Abb. 2, 3), b Sagittale Rekonstruktion links mit intakter Fissur zwischen Ober- und Unterlappen. 

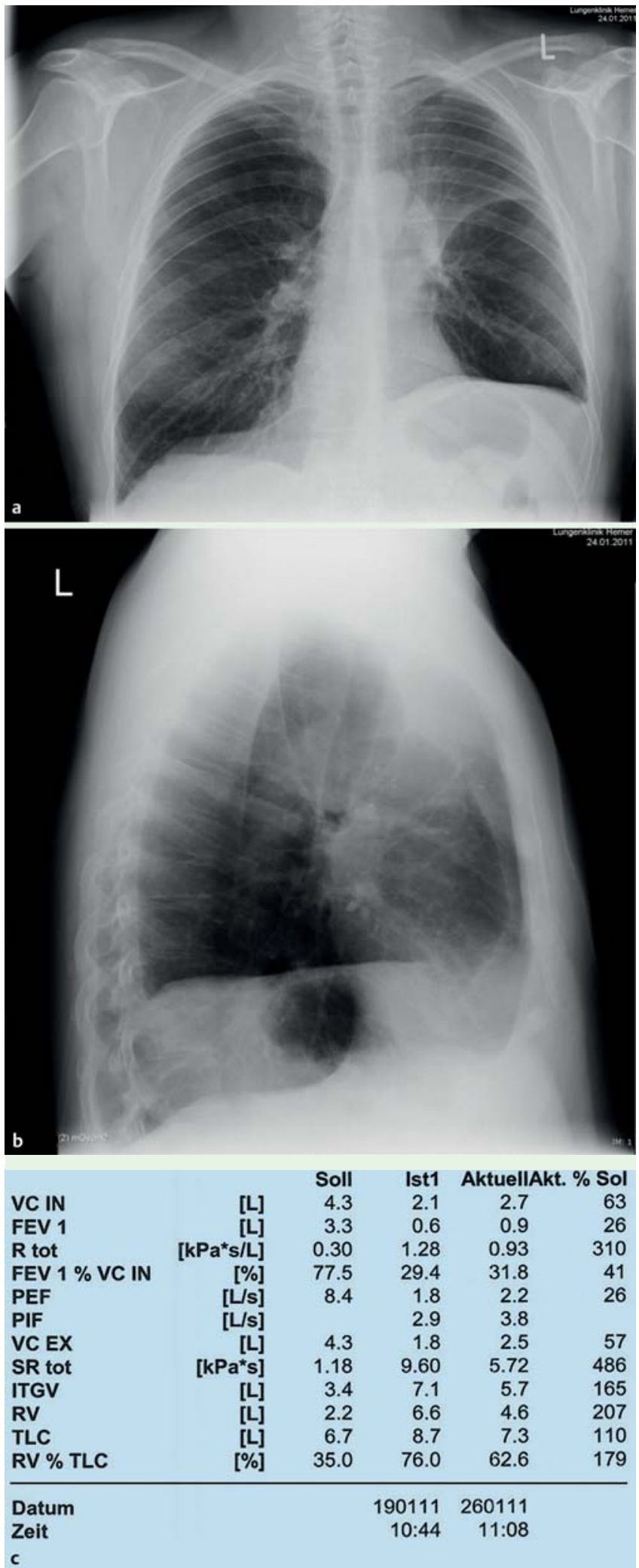

Abb. 6 a Oberlappenatelektase links nach Implantation von 4 IBV (Spira$\operatorname{tion}^{\circledR}$ ). Beachte den deutlichen Volumenreduktionseffekt mit Hochstand des Zwerchfells. b In der Seitaufnahme Umbrellaphänomen bei Oberlappenatelektase links. Zentral liegende Ventile (Spiration ${ }^{\circledR}$ ), c Dazugehörige Lungenfunktionskontrolle. Ist1 entspricht dem Zustand vor Ventileinlage, Aktuell dem Zustand nach Ventileinlage. Beachte den Anstieg von $\mathrm{FEV}_{1}$ um $50 \%$, den Anstieg von VC IN um 0,6 L und den Abfall des RV um 2,0 L.

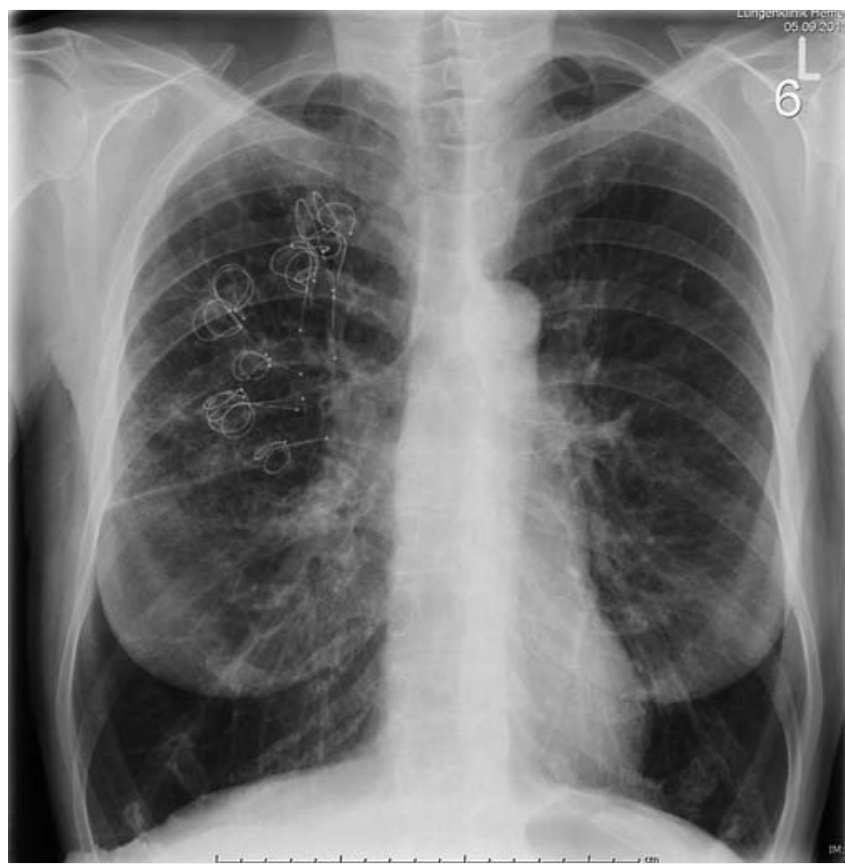

Abb.7 Nach Einlage von 10 Coils (PneumRx ${ }^{\circledR}$ ) in den rechten Oberlappen (identische Pat. wie Abb. 2, 3, 5).

Trägerkatheter über den Biopsiekanal des Fiberbronchoskops in einen Segment- bzw. Subsegmentbronchus eingebracht. Nach der Freisetzung aus dem Trägerkatheter nehmen diese wieder die Spiralform an und verformen Bronchien wie angrenzende Lunge und damit den gesamten behandelten Lappen $(\bullet$ Video 2$)$. Meist werden etwa 10 Coils für einen Oberlappen verwendet $(\bullet$ Abb. 7). Neben der Verformung spielt wahrscheinlich eine teilweise Blockade des Bronchus eine Rolle. Die Coils werden bei heterogenem oberlappenbetontem Emphysem verwandt. Der Vorteil im Vergleich zu den Ventilen ist, dass dieses Verfahren unabhängig von kollateraler Ventilation ist. Damit kann es bei Patienten eingesetzt werden, die aufgrund inkompletter Fissuren oder nachgewiesener kollateraler Ventilation nicht für Ventile geeignet erscheinen.

Bislang sind zu diesen Verfahren nur kleinere Studien publiziert. Eine erste Pilotstudie [19] bei 11 Patienten mit homogenem und heterogenem Emphysem zeigt eine geringe Verbesserung bei Lungenfunktionsparametern, im 6-Minuten-Gehtest und in einem Befindlichkeitsindex. In einer Subgruppenanalyse zeigt sich beim heterogenen Emphysem eine höhere Erfolgsrate. In einer prospektiv angelegten Folgestudie bei Patienten mit heterogenem Lungenemphysem an 16 Patienten konnte eine signifikante Verbesserung der Lungenfunktion, der Belastbarkeit und der Lebensqualität erreicht werden [20]. Die Coils können im Gegensatz zu den Ventilen meist nicht oder nur sehr schwer entfernt werden. Das proximale Ende der Coils ist nur selten sichtbar und

\section{Video 2}

Bereits mehrere Coils im rechten Oberlappen erkennbar. Über den im Bronchus liegenden Katheter wird ein Coil in den Oberlappen bis zur Markierung vorgeschoben und durch Rückzug des Katheters freigesetzt. Es nimmt dann seine Spiralform an.

\section{Video unter:}

www.thieme-connect.de/ejournals/doi/10.1055/s-0031-1291458 


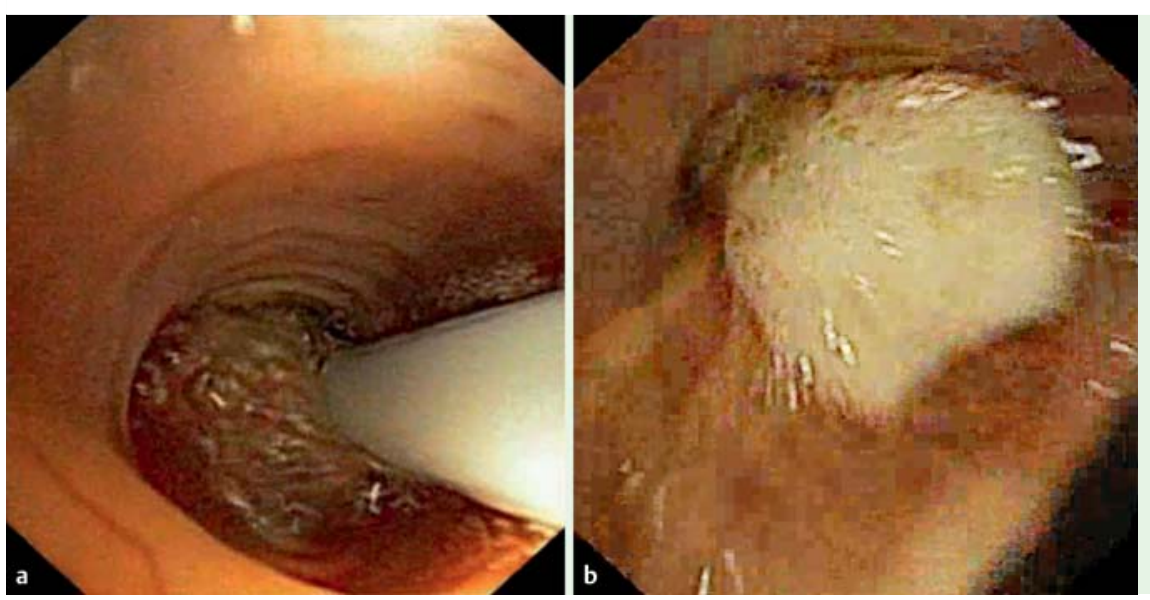

Abb. 8 a Instilliertes AeriSeal ${ }^{\circledR}$ mit noch sichtbarem Applikationskatheter. b Unmittelbar nach Applikation von AeriSeal ${ }^{\circledR}$ verbleibender Clot im Segmentbronchus.

erreichbar. Zudem kommt ein Zeitfaktor hinzu. Eine Entfernung wird nur in den ersten Wochen nach Implantation empfohlen, weil die Coils tief im Gewebe verankert werden. Damit muss das Verfahren als weitgehend irreversibel eingeordnet werden.

Die nicht blockierenden Verfahren umfassen die polymerische Lungenvolumenreduktion [21] und die thermische Ablation. Sie sind irreversibel. Bei der polymerischen Lungenvolumenreduktion (PLVR, Aeris Therapeutics, USA) wird ein Hydrogel-Schaum (AeriSeal ${ }^{\circledR}$ ) in zerstörte Subsegmente der Lunge appliziert ( Abb.8, Video 3). Der Mechanismus ist neben einer induzierten Entzündung und Schrumpfung des Lungenabschnitts die Versiegelung des Abschnitts durch das adhäsiv wirkende Polymer mit einer Verstärkung des Effekts durch Resorption der Luft. Zur PLVR sind bereits mehrere Studien publiziert. Eine Studie an 50 Patienten mit oberlappenbetontem Emphysem zeigte 3 Monate nach Applikation des Polymers eine signifikante Verbesserung von $\mathrm{FEV}_{1}$ und RC/TLC [22]. Bei Behandlung von mehreren Subsegmenten wird ein größerer Effekt erzielt, allerdings zu Lasten einer gesteigerten inflammatorischen Reaktion [23]. Weitere Daten zeigen, dass bei Patienten mit COPD im Stadium III bessere Ergebnisse erzielt werden als bei Patienten im Stadium IV [9]. Zudem ist eine polymerische Lungenvolumenreduktion auch beim homogenen Emphysem möglich, allerdings sollte dann eine größere Anzahl von Subsegmenten behandelt werden [23].

Die bronchoskopische Thermoablation basiert auf der Applikation von heißem Wasserdampf über einen flexiblen geblockten Katheter über den Arbeitskanal eines Fiberskops in einen definierten Lungenabschnitt, meist ein Lungensegment. Ein inflammatorischer Reiz erzeugt dort eine akute Gewebsverletzung, gefolgt von Vernarbung, Fibrosierung und Schrumpfung. Dieses Verfahren wurde bei 11 Patienten mit heterogenem Emphysem eingesetzt. Dabei wird der gesamte Oberlappen therapiert. Es ergibt sich eine signifikante Verbesserung des $\mathrm{FEV}_{1}$, des 6-MinutenGehtests und der Lebensqualität [24,25].

\section{Video 3}

Der Applikationskatheter wird bis über die schwarze Markierung hinaus in den Subsegmentbronchus vorgeschoben. Das Polymer wird über diesen eingebracht. Nach Entfernen des Katheters verbleibt zunächst ein Clot.

Video unter:

www.thieme-connect.de/ejournals/doi/10.1055/s-0031-1291458

\section{Alternativen}

Zur Behandlung des homogenen Emphysems ist die Schaffung von Atemwegskurzschlüssen (Broncus Technologies, USA) im Rahmen einer multizentrischen Studie untersucht. Über diese Kurzschlüsse soll gefesselte Luft entweichen und die Überblähung reduziert werden. Diese Kurzschlüsse werden von den zentralen Bronchien ins Lungengewebe mithilfe einer Nadelperforation geschaffen, dilatiert und durch einen medikamentenbeschichteten Stent gesichert. Bei 315 Patienten wurde dieses Verfahren im Rahmen einer doppelblinden, randomisierten multizentrischen Studie überprüft. Das Ergebnis ist insgesamt enttäuschend. Der frühe nachweisbare Effekt einer signifikanten Lungenvolumenreduktion ist nur kurzfristig vorhanden. Bereits nach 6 Monaten kann kein Unterschied zwischen den Armen mehr nachgewiesen werden [26], vermutlich durch einen Verschluss der geschaffenen Nebenwege. Deshalb hat sich das Verfahren nicht durchsetzen können. Die Schaffung eines transthorakalen Stomas zur Ableitung stellt ebenfalls ein Verfahren dar, das beim homogenen Emphysem zum Einsatz kommt (PortAero, USA). Hierzu sind aber keine publizierten Daten verfügbar.

\section{Zusammenfassung \\ $\nabla$}

Die ELVR umfasst diverse wenig invasive Verfahren, deren Sicherheit und Effektivität durch eine ganze Reihe von Publikationen belegt ist. Die Ergebnisse sind zum Teil vielversprechend. Bei einzelnen Patienten werden erhebliche Verbesserungen erreicht, zum Teil sind die Ergebnisse aber enttäuschend. Ein weiterer Nachteil ist das weitgehende Fehlen von Daten zum Langzeitverlauf. Der Schwerpunkt der aktuellen Forschung liegt in der Identifikation geeigneter Subgruppen, die langfristig von den verschiedenen Verfahren profitieren. Die ELVR ist noch insgesamt zurückhaltend zu bewerten, bis durch weitere Studien die offenen Fragen geklärt sind [27].

\section{Interessenkonflikt}

$\nabla$

Der Autor gibt an, dass kein Interessenkonflikt besteht. 


\section{Literatur}

1 Hopkinson NS, Kemp SV, Toma TP et al. Atelectasis and survival after bronchoscopic lung volume reduction for COPD. Eur Respir J 2011; 37: $1346-1351$

2 Geddes D, Davies $M$, Koyama $H$ et al. Effect of lung-volume reduction surgery in patients with severe emphysema. N Engl J Med 2000; 343: 239-245

3 National Emphysema Treatment Trial Research Group. A randomized trial comparing lung-volume-reduction surgery with medical therapy for severe emphysema. N Engl J Med 2003; 348: 2059-2073

4 Ferguson GT, Fernandez E, Zamora MR et al. Improved exercise performance following lung volume reduction surgery for emphysema. Am J Respir Crit Care Med 1998; 157: 1195-1203

5 Ramsey SD, Shroyer AL, Sullivan SD et al. Updated evaluation of the costeffectiveness of lung volume reduction surgery. Chest 2007; 131: $823-832$

6 Naunheim KS, Wood DE, Mohsenifar Z et al. National Emphysema Treatment Trial Research Group. Long-term follow-up of patients receiving lung-volume-reduction surgery versus medical therapy for severe emphysema by the National Emphysema Treatment Trial Research Group. Ann Thorac Surg 2006; 82: 431-443

7 Sciurba FC, Ernst A, Herth FJF et al. A randomized study of endobronchial valves for advanced emphysema. New Engl J Med 2010; 363: 1233 1244

8 Hopkinson NS. Bronchoscopic lung volume reduction: indications, effects and prospects. Curr Opin Pulm Med 2007; 13: 125-130

9 Herth FJF, Gompelmann D, Stanzel F et al. Treatment of advanced emphysema with emphysematous lung sealant $\left(\right.$ AeriSeal $\left.^{\circledR}\right)$. Respiration 2011; 82: 36-45

10 Kuhnigk JM, Dicken V, Zidowitz $S$ et al. New tools for computer assistance in thoracic CT. Part 1. Functional analysis of lungs, lung lobes, and bronchopulmonary segments. RadioGraphics 2005; 25: 525-536

11 Toma TP, Polkey MI, Goldstraw PG et al. Methodological aspects of bronchoscopic lung volume reduction with a proprietary system. Respiration 2003; 70: 658-664

12 Toma TP, Hopkinson NS, Hillier J et al. Bronchoscopic volume reduction with valve implants in patients with severe emphysema. Lancet 2003; 361: 931 - 933

13 Hopkinson NS, Toma TP, Hansell DM et al. Effect of bronchoscopic lung volume reduction on dynamic hyperinflation and exercise in emphysema. Am J Respir Crit Care Med 2005; 171: 453-460
14 Gompelmann D, Eberhardt R, Michaud G et al. Predicting atelectasis by assessment of collateral ventilation prior to endoscopic lung volume reduction: a feasibility study. Respiration 2010; 80: 419-425

15 Wood DE, McKenna RJJr, Yusen RD et al. A multicenter trial of an intrabronchial valve for treatment of severe emphysema. J Thorac Cardiovasc Surg 2007; 133: $65-73$

16 Sterman DH, Mehta AC, Wood D et al. IBV valve pilot trial research team. A multicenter pilot study of a bronchial valve for the treatment of severe emphysema. Respiration 2010; 79: 222 -233

17 Springmeyer SC, Bolliger CT, Waddell TK et al. Treatment of heterogeneous emphysema using the spiration IBV valves. Thorac Surg Clin 2009; 19: 247-253

18 Eberhardt R, Gompelmann D, Schuhmann M et al. Unilateral vs. bilateral endoscopic lung volume reduction in patients with severe heterogenous emphysema: A comparative randomised case study. ERS; 2010

19 Herth FJF, Eberhardt R, Gompelmann D et al. Bronchoscopic lung volume reduction with a dedicated coil: a clinical pilot study. Ther Adv Respir Dis 2010; 4: 225-231

20 Slebos DJ, Kerstjens HAM, Ernst $A$ et al. Bronchoscopic lung volume reduction coil treatment of severe heterogeneous emphysema. ERS; 2010

21 Ingenito EP, Berger RL, Henderson AC et al. Bronchoscopic lung volume reduction using tissue engineering principles. Am J Respir Crit Care Med 2003; 167: $771-778$

22 Criner GJ, Pinot-Plata V, Strange $C$ et al. Biologic lung volume reduction in advanced upper lobe emphysema: Phase 2 results. Am J Respir Crit Care 2009; 179: 791 - 798

23 Refaely Y, Dransfield M, Kramer MR et al. Biologic lung volume reduction therapy for advanced homogeneous emphysema. Eur Respir J 2010; 36: 20-27

24 Herth FJF, Schmidt B, Ficker J et al. Germany pilot safety and feasibility study of bronchoscopic thermal vapor ablation (BTVA) for lung volume reduction in patients with heterogenous emphysema with upper lobe predominance. Eur Resp J 2009; 35: 1827

25 Snell GI, Hopkins P, Westall G et al. A feasibility and safety study of bronchoscopic thermal vapor ablation: a novel emphysema therapy. Ann Thorac Surg 2009; 88: 1993 - 1998

26 Shah PL, Slebos DJ, Cardoso PFG et al. EASE trial study group. Bronchoscopic lung-volume reduction with Exhale airway stents for emphysema (EASE trial): randomised, sham-controlled, multicentre trial. Lancet 2011; 378: $997-1005$

27 Herth FJF, Gompelmann D, Ernst A et al. Endoscopic lung volume reduction. Respiration 2010; 79: 5-13 